\title{
Bioengineering Cartilage Growth, Maturation, and Form
}

\author{
GREGORY M. WILLIAMS, STEPHEN M. KLISCH, AND ROBERT L. SAH
}

\begin{abstract}
Department of Bioengineering [G.M.W., R.L.S.], Whitaker Institute of Biomedical Engineering [R.L.S.], University of California, San Diego, California 92093; Department of Mechanical Engineering [S.M.K.], California Polytechnic State University, San Luis Obispo, California 93407
\end{abstract}

\begin{abstract}
Cartilage of articular joints grows and matures to achieve characteristic sizes, forms, and functional properties. Through these processes, the tissue not only serves as a template for bone growth but also yields mature articular cartilage providing joints with a low-friction, wear-resistant bearing material. The study of cartilage growth and maturation is a focus of both cartilage biologists and bioengineers with one goal of trying to create biologic tissue substitutes for the repair of damaged joints. Experimental approaches both in vivo and in vitro are being used to better understand the mechanisms and regulation of growth and maturation processes. This knowledge may facilitate the controlled manipulation of cartilage size, shape, and maturity to meet the criteria needed for successful clinical applications. Mathematical models are also useful tools for quantitatively describing the dynamically changing composition, structure and function of cartilage during growth and maturation and may aid the development of tissue engineering solutions. Recent advances in methods of cartilage formation and culture which control the size, shape, and maturity of these tissues are numerous and provide contrast to the physiologic development of cartilage.

(Pediatr Res 63: 527-534, 2008)
\end{abstract}

$\mathrm{F}$ ollowing the formation of a synovial joint, cartilage growth and maturation allow for the enlargement of the joint and attainment of the adult properties allowing the cartilage to function for many decades as a low friction, wear resistant, bearing material. However, injury and degenerative conditions of the articular cartilage may compromise these functions and result in pain and disability. The treatment paradigm for articular cartilage damage frequently involves surgical intervention, since mature cartilage has a poor capacity for repair. Surgical methods range from penetrating the subchondral bone to elicit a repair response to implantation of cells and tissues to the replacement of partial or whole joints (1). Cartilage tissue engineering has been an active area of study focused on bringing biologically-active tissue substitutes into clinical use. Tissue engineering strategies offer the potential to provide benefits beyond those of the current repair procedures, including the formation of more consistent hyaline-like repair tissue, the use of nonimmunogenic cells and

Received December 10, 2007; accepted January 16, 2008

Correspondence: Robert L. Sah, M.D., Sc.D., University of California, San Diego, Whitaker Institute of Biomedical Engineering, Department of Bioengineering, 9500 Gilman Dr., Mail Code 0412, La Jolla, CA 92093; e-mail: rsah@ucsd.edu

This work was supported by grants from the National Institutes of Health (to SMK and RLS), the National Science Foundation (to SMK and RLS), and the Howard Hughes Medical Institute through the HHMI Professors Program (to the University of California, San Diego, in support of RLS) and a National Science Foundation Graduate Research Fellowship (to GMW). tissues, enhanced integration with surrounding tissues, and reduced rehabilitation times. The ability to realize these advances will likely depend equally on knowledge of cartilage biology and engineering innovations.

The diversity of form and functional properties in the body's many joints arises from the genetic, epigenetic, and environmental factors which govern cartilage morphogenesis, growth, and maturation. Disruptions of these regulating factors, occurring spontaneously or in a controlled manner in vivo or in vitro, can alter the normal course of cartilage development and growth. Each of these occurrences serves as an opportunity to learn about the mechanisms underlying these phenomena and apply that knowledge to the treatment of cartilage diseases and injuries.

The goal of this article is to review aspects of articular and epiphyseal cartilage growth, maturation, and morphogenesis with applications toward bioengineering chondral tissue substitutes for therapeutic purposes. A thorough review of the cartilage developmental biology is not intended, since a number of good reviews of the subject already exist (2-4). However, the lessons learned from cartilage developmental biology are important to understanding and improving the in vitro engineering of cartilaginous tissues. These translational aspects of cartilage biology as they relate to bioengineering analysis and cartilage tissue engineering will emerge as the focus of this article.

Cartilage growth, remodeling, maturation, and morphogenesis typically occur concurrently during the course of in vivo joint development, yet these processes might be manipulated independently in an in vitro system. Some definitions are provided here to clarify the meaning of these terms, as used herein. Although the term growth may be used loosely to encompass many of the changes in a tissue before reaching maturity, a more rigorous interpretation is useful for bioengineering analyses. Simply, growth is the increase in tissue volume resulting from the accumulation of material similar to the original. As is often the case, growth may be accompanied by remodeling of the existing tissue to incorporate the new material. However, remodeling, which refers to the modification of tissue properties via biochemical and biophysical changes in structure, may also occur in the absence of growth $(5,6)$. Maturation is the progressive change of tissue composition and structure to achieve the functional properties of the

\footnotetext{
Abbreviations: BMP, bone morphogenetic protein; DDH, developmental dysplasia of the hip; GAG, glycosaminoglycan
} 
adult state. Last, morphogenesis is the development of form resulting from specific patterns of cellular organization and tissue growth and remodeling during embryogenesis (7). With these underlying definitions, it is possible to exam cartilage growth, maturation, and morphogenesis using both physical and mathematical approaches.

\section{GROWTH AND MATURATION}

Mechanisms and modes of growth. The growth of cartilage may arise nonuniformly in distinct regions of the tissue through specific biologic activities of the indwelling cells termed chondrocytes. These cells are embedded in a highly hydrated extracellular matrix consisting predominantly of collagen type II and aggrecan, a large, negatively charged proteoglycan (8). Growth may be mediated through increases in either the cell volume fraction by proliferation or hypertrophy or in the matrix volume fraction by a net accretion of constituent molecules. Early in cartilage development, the tissue is highly cellular, so significant changes in cell number or volume would likely have proportional effects on the tissue volume. As sources of matrix secretion, chondrocytes at high density would also be well poised to contribute to growth through their anabolic production of matrix molecules. However at later stages of growth, chondrocyte density is reduced (9), and the capacity for further growth is diminished.

The growth of a tissue may also occur in two distinct modes: growth throughout the volume of the substance, termed interstitial growth, and growth at a surface or interface, termed appositional growth (10). Interstitial growth is simply illustrated as a spheroidal material occupying region $R_{1}$ at time $t=t_{1}$ which grows throughout the volume to occupy region $\mathrm{R}_{2}$ at $t=t_{2}$ (Fig. 1A). Assuming that the set of material points in $R_{1}$ is dense in the set of points in $R_{2}$, points in $R_{2}$ (e.g., $\mathrm{P}_{2}$ and $\mathrm{Q}_{2}$ ) can be mapped back to points in $\mathrm{R}_{1}\left(\right.$ e.g., $\mathrm{P}_{1}$ and $\mathrm{Q}_{1}$ ) (11). Similarly, appositional growth is illustrated as

\section{A Interstitial Growth}
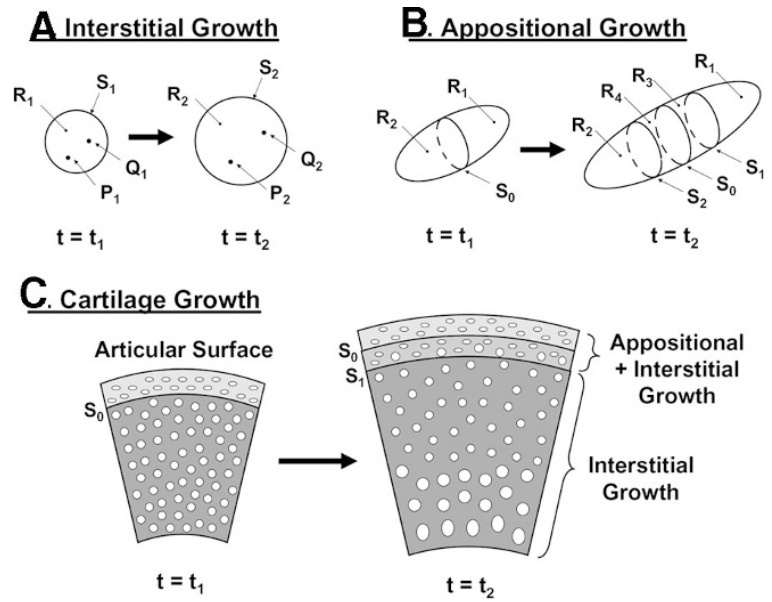

Figure 1. (A) Interstitial growth is illustrated as a material occupying region $\mathrm{R}_{1}$ at $t=t_{1}$ and $\mathrm{R}_{2}$ at $t=t_{2}$. (B) Two-sided appositional growth at surface $\mathrm{S}_{0}$ results in no growth within regions $\mathrm{R}_{1}$ and $\mathrm{R}_{2}$, but formation of new regions $\mathrm{R}_{3}$ and $\mathrm{R}_{4}$. (C) Cartilage growth potentially includes both interstitial growth by matrix accretion and chondrocyte hypertrophy and appositional growth by proliferation of cells in the superficial-most layer demarcated by $\mathrm{S}_{0}$. Illustrations in (A) and (B) are adapted, from Skalak R et al., J Theor Biol 94:555-577, Copyright (C) 1982 Elsevier, Ltd., with permission. an ellipsoidal material with a two-sided growth surface, $\mathrm{S}_{0}$, separating material occupying regions $\mathrm{R}_{1}$ and $\mathrm{R}_{2}$ at $t=t_{1}$ (Fig. 1B). New material is deposited on both sides of the growth surface forming regions $\mathrm{R}_{3}$ and $\mathrm{R}_{4}$ by $t=t_{2}$, whereas the original regions $\mathrm{R}_{1}$ and $\mathrm{R}_{2}$ might deform only to accommodate these new regions.

Examining which biologic mechanisms of growth are active in the different regions of cartilage is critical to determining its modes of growth. Using tritiated thymidine, two bands of proliferating chondrocytes were identified in immature rabbit knees, one near the articular surface and a second, deeper and close to the ossification front (12). By detecting proliferating cell nuclear antigen or the incorporation of bromodeoxyuridine, the presence of a proliferating layer of cells at the articular surface in immature rats, bovines, and South American possums was confirmed, though these studies did not find a deeper band of dividing cells $(13,14)$. This thin layer of proliferating cells near the surface may serve as a means of appositional growth (15), which is consistent with the reported existence of a progenitor cell population at the articular surface (16-18). However, the lack of mitotic activity in the bulk of the growing cartilage suggests that cell division plays little or no role in interstitial growth of articular cartilage.

Interstitial growth of cartilage is likely to arise from the enlargement of chondrocytes and their robust production of matrix molecules. In the growth plates of rats, these two processes result in much of the total tissue elongation, with chondrocyte hypertrophy accounting for $44-59 \%$ of the total growth and matrix accretion accounting for $32-49 \%$ (19). Similar quantification of the contribution of these processes to overall tissue growth in epiphyseal cartilage is lacking, though there is evidence that both occur. Increasing chondrocyte size with depth in mature articular cartilage lends support to the contribution of hypertrophy (20). Chondrocyte density also decreases significantly with growth and maturation, which in the absence of appreciable cell death is indicative of abundant matrix accretion (9). Thus, cartilage growth may proceed via a combination of appositional and interstitial growth (Fig. $1 C)$. Understanding these modes of growth and the underlying biologic processes may facilitate tissue engineering efforts to grow cartilage of a required size.

Changes accompanying maturation. The maturation of cartilage in vivo is coincident with the growth of the tissue and can be characterized by a number of changes in the tissue's structure, composition, and functional properties. The bovine stifle joint provides a well-studied example of articular cartilage properties at various stages of maturity (third trimester fetal, 1-3 wk-old calf, and 1-2-y-old young adult). As mentioned previously, cellularity decreases with maturation from fetus to adult by roughly half or more $(9,21,22)$. The solid content (dry weight/tissue volume) increases from $\sim 12 \%$ in the fetus to $\sim 18 \%$ in the calf and $\sim 20 \%$ in the adult (21). This change in solid content is largely attributable to an increasing collagen concentration which may nearly triple from fetus to adult $(21,22)$. During this period the collagen network is also stabilized through the formation of pyridinoline crosslinks, which may increase in concentration from the fetal stage by 2to 4-fold in the calf and greater than 7-fold in the adult 
$(22,23)$. On the other hand, the glycosaminoglycan (GAG) concentration is relatively maintained $(21,22)$, though the size of individual chondroitin sulfate chains and their ratio to keratan sulfate typically decrease with maturation (24). These compositional changes from fetus to adult are manifested in marked alterations of the cartilage mechanical properties including a $\sim 180 \%$ increase in compressive aggregate modulus and a $\sim 450 \%$ increase in equilibrium tensile modulus $(21,22)$. A large portion of these biochemical and functional changes takes place in the few short weeks between the late fetal and neonatal periods. This demonstration of rapid maturation during normal growth supports the concept that under certain conditions the functional properties of engineered cartilage may be significantly augmented during brief in vitro culture or during a short, in vivo rehabilitation time.

Cartilage maturation is also reflected by the architecture of the tissue. The thicker immature cartilage is marked by features which identify it as both articular cartilage and epiphyseal growth plate. The bulk of the immature cartilage is often described as having a fairly isotropic structure with chondrocytes arranged at random $(25,26)$. However, cell density may decrease with depth, particularly near the articular surface (25), whereas GAG and collagen concentrations and tensile and compressive moduli may increase $(27,28)$. On the other hand, mature articular cartilage possesses distinct superficial, middle, and deep zones. In mature tissue, this transition, from the superficial most layer to the deepest, is characterized by cellular changes including a decrease in density, a rounding of morphology, and an organization into clusters in the middle zone and columns in the deep zone $(25,26)$. Likewise, aggrecan content increases with depth (29), and the predominant collagen orientation shifts from parallel to perpendicular to the articular surface (30). These compositional and structural differences are also reflected in the depth-varying mechanical properties in compression and tension $(31,32)$. Mature articular cartilage also bears the distinguishing feature of a calcified cartilage interface with the subchondral bone. This feature provides a strong interdigitated bond between uncalcified cartilage and bone through which joint forces are transmitted. The formation of similar architectural features in engineered tissues may provide mechanical and tribological properties that are important for successful joint repair.

Mathematical descriptions of growth. Complementing the physiologic aspects of growth, mathematical descriptions of growing bodies can provide a framework in which growth phenomena can be analyzed. General approaches for analytically describing both volumetrically distributed (interstitial) growth and surface (appositional) growth have been summarized previously (10). Volumetrically distributed growth may be modeled using a growth extension tensor analogous to a material strain tensor in solid mechanics, while surface growth may be represented through a velocity field at the growth surface (10). These fundamental growth models are widely adaptable to better understand any number of growing tissues including cartilage and bone.

Expanding upon the volumetrically distributed growth model, cartilage growth mixture models have been developed using a continuum mechanics approach $(5,6)$. In these models, cartilage is represented as a mixture of components: typically two growing elastic constituents, namely proteoglycans and collagen, and an inviscid fluid. Other cartilage constituents, such as collagen crosslinks or cells, may be included, since the theory allows for an arbitrary number of growing components. The process of growth is decomposed into two separate tensors which describe growth deformations from mass deposition and mechanical deformations which provide compatibility of the constituents in an equilibrium stress state. Changes in the size, biochemical composition, and tensile properties of growing cartilage explants in vitro have been successfully described using this growth mixture model approach (6). With similar assumptions, a cartilage growth finite element model has been proposed recently and may facilitate the description of specific experimental protocols such as compression or perfusion (33). Such models of cartilage growth may ultimately be useful for predicting the geometry, composition, and biomechanical properties of a tissue.

Compartmental and continuum metabolic models of cartilage growth and remodeling provide an alternative means of describing changing tissue composition. Metabolic models are based on the principle of mass balance for one or more components such as proteoglycan or collagen. Single compartment models have been frequently used in the analysis of radiolabel experiments to determine rates of molecular synthesis and degradation. These models have been used to quantify the effects of chemical and mechanical stimuli on the metabolism of cartilage proteoglycans and collagens (34-36). The kinetics of collagen crosslinking in cartilage explants has also been described using similar compartmental models (37). Although a compartmental model can only account for the spatially averaged concentration of a component over time, a continuum model may describe the spatial distribution of such a component. One such continuum model was developed with consideration of the synthesis, transport, binding, and degradation of matrix components and was applied to understanding proteoglycan distributions in articular cartilage (38). Recently, this model was expanded to analyze the effects of fluid flow and membrane permeability on the development of depth varying composition of engineered cartilage in a perfusion bioreactor (39). Together, these types of models of cartilage growth and metabolism help provide a quantitative description of the dynamics of growth, remodeling, and maturation which may be useful in creating or evaluating engineered tissues.

In vivo regulation of growth and maturation. Chondrocyte cell fates and metabolic activities provide the means of cartilage growth and maturation and are regulated by chemical and mechanical stimuli (Fig. 2). Applied stimuli are transmitted through the tissue according to the physical and chemical properties and become a chemical or mechanical change in the chondrocyte microenvironment. These microenvironmental signals potentially act upon the chondrocytes to alter cell fate and matrix metabolism. In turn, these cellular activities may change the composition, structure, and functional properties of the tissue, thereby modifying the way subsequent stimuli are perceived. Determining what stimuli regulate cartilage growth and maturation and by what mechanisms they act is critical to 


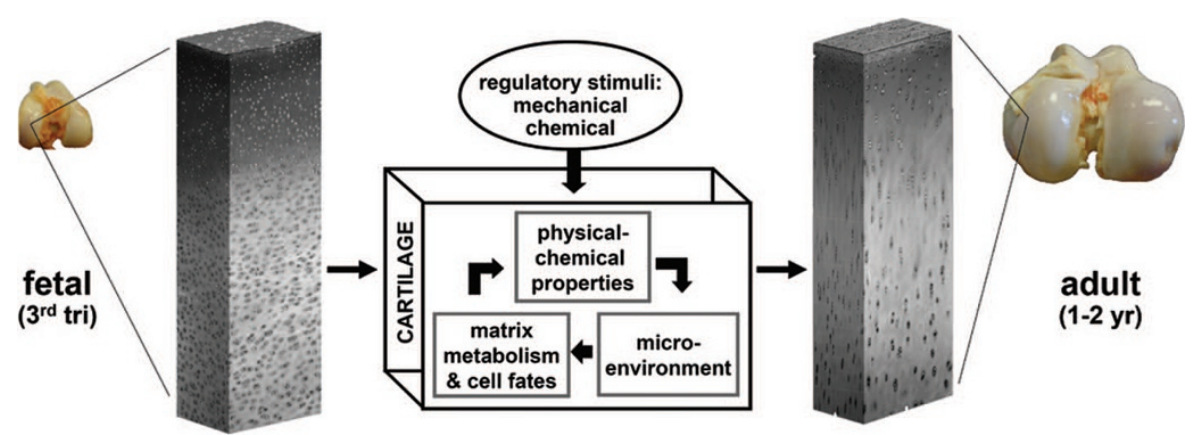

Figure 2. Dynamic processes govern the growth and maturation of articular cartilage as shown for the fetal to adult transition of the bovine knee. Regulatory stimuli are transformed according to the physical and chemical properties of the tissue into signals in the chondrocyte microenvironment to alter matrix metabolism and cell fates. These cellular processes, in turn, are responsible for the enlargement of the joint and the evolving tissue composition, structure, and functional properties. Some of these changes, particularly the decrease in cell density and attainment of a mature, stratified architecture, are demonstrated by cartilage digital volumetric images. Reprinted from Jadin KD et al., J Histochem Cytochem 53:1109-1119, Copyright @ 2005 The Histochemical Society, Inc., with permission.

understanding cartilage development and may be applicable to the engineering of biologic tissue substitutes.

A large effort has been put forth to identify the molecular mediators such as growth factors and transcription factors which govern joint formation, growth, and maturation. Regulatory molecules from various growth factor families such as the insulin-like growth factors, fibroblast growth factors, and transforming growth factors- $\beta$ (TGF- $\beta$ ), which includes the bone morphogenetic proteins (BMP) and growth differentiation factors, have been implicated in these processes. Many human skeletal defects are associated with mutations in genes encoding these growth factors as well as their receptors and antagonists, a selection of which is nicely summarized by Shum et al. (2). The development of transgenic mice has provided great insight into how these chemical regulators affect cartilage development. However, many of the chemical signaling molecules which influence cartilage growth and maturation have functions earlier in development, for example in cellular differentiation or joint site patterning. The disruption of these earlier functions can mask the effects of these molecules in later stages of growth. In such cases, the conditional inactivation of the gene in cartilage at a specified time may help clarify the roles of these signaling molecules.

Mechanical stimuli also serve a regulatory function for cartilage growth and maturation in vivo. Joint morphology, which is the physical manifestation of spatially nonuniform cartilage growth, is sensitive to the mechanical environment of the joint. Although further detail will be given in a later section, it is important to note that abnormal cartilage growth and joint morphology may result from altered physical forces on the tissue during prenatal development and beyond. In addition, a number of in vivo studies with immature dogs have provided evidence that different regimes of joint loading, via exercise or limb immobilization, may lead to significant differences in articular cartilage structure (e.g., thickness), composition (e.g., proteoglycan content), and functional properties (e.g., indentation stiffness) (40).

In vitro manipulation of growth and maturation. The culture of isolated cells or cartilage explants in a controlled in vitro environment has yielded additional insight into the biochemical regulation of cartilage growth and maturation. Although the processes of growth and maturation proceed con-

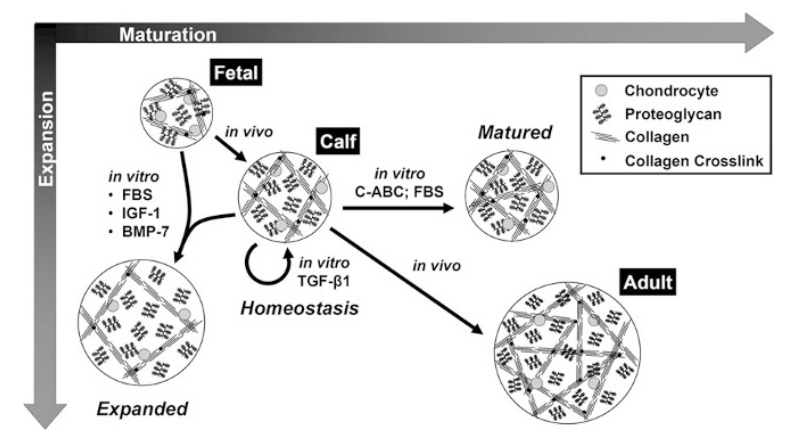

Figure 3. Expansion (or size), maturation (e.g., tensile or compressive stiffness) and composition are properties of cartilage controlled by the application of regulatory stimuli. In vivo, cartilage grows and matures concurrently; however, expansion and maturation may be manipulated independently within a controlled in vitro environment. Explants of immature cartilage cultured with fetal bovine serum (FBS), insulin-like growth factors, or bone morphogenetic protein-7 (BMP-7) exhibit expansive growth, while those pretreated with chondroitinase $\mathrm{ABC}(\mathrm{C}-\mathrm{ABC})$ followed by culture in FBS undergo maturational growth. Culture in transforming growth factor- $\beta 1$ (TGF- $\beta 1$ ) yields a near homeostatic state.

currently in vivo, an in vitro system may allow for independent manipulation of these processes (Fig. 3). It has been hypothesized that changes in cartilage size and in maturation (as quantified by the tensile properties) could result by altering the balance of proteoglycan and collagen metabolism $(27,41,42)$. Culturing immature cartilage explants with fetal bovine serum (FBS), insulin-like growth factors-1, or bone morphogenetic protein-7 (BMP-7) resulted in an expansive growth phenotype characterized by large increases in size, stimulation of proteoglycan in excess of collagen, and decreases in tensile stiffness and strength (42). Alternatively, culture with TGF- $\beta 1$ promoted cartilage homeostasis with little change in size, composition, or mechanical properties (42). The identification of biochemical stimuli which promote maturation in immature cartilage explants has been elusive, though depletion of proteoglycans by chondroitinase-ABC treatment before culture resulted in increased tensile stiffness (27). These distinct treatments to promote expansion, homeostasis, or maturation may find use in tissue engineering cartilage of specific size or maturity.

In vitro experiments have also been useful for examining the effects of mechanical stimuli, such as static and dynamic 
compression, hydrostatic pressure, and fluid shear, on the metabolic activities, particularly those of aggrecan, in cartilage explants and cells (43). The application of such stimuli in a controlled manner in vitro provides an accurate knowledge of the loading history, though these simplified loading conditions do not fully reflect the complexities of joint loading and articulation. The effects of loading on metabolism are dependent on the magnitude and frequency of loading, with the general paradigm that static stresses inhibit aggrecan synthesis while moderate dynamic stresses promote synthesis.

Enhancement of engineered cartilage composition and mechanical properties may also be prescribed through specific regimes of biochemical and mechanical stimuli. The effects produced by biochemical stimuli are dependent on magnitude (or dose) and frequency (duration and timing) just like their mechanical counterparts. Further complexity arises from the possibility of additive and synergistic effects of combinations of different stimuli. As an example, one recent study reports that sequential stimulation of agarose-chondrocyte constructs with TGF- $\beta 3$ followed by dynamic compressive loading resulted in significantly higher compressive modulus and GAG content compared with constructs which received the same growth factor stimulus but were mechanically stimulated or left free-swelling throughout the culture (44). That study and others have been able to produce engineered cartilage containing proteoglycans at or near native levels following weeks or months of culture $(44,45)$. However, engineering cartilage with collagen contents approaching native levels has proved to be more challenging. This problem is made more difficult by the rapidly changing tissue dynamics illustrated in Figure 2. As is often the case, engineered cartilage consists of cells elaborating a matrix into a nonnative environment, such as a scaffold, hydrogel, or high-density culture. The different physicochemical properties, initial lack of cell-matrix interactions, and altered cell morphology may cause cells in engineered tissues to respond to stimuli in a manner unlike that of chondrocytes in their native extracellular matrix (46-48).

Engineering the architecture of mature cartilage. The zonal stratification of articular cartilage is a hallmark of maturation and the recapitulation of this architecture has been a focus of recent tissue engineering efforts. Several studies have taken advantage of the phenotypic differences of chondrocytes from different zones of native cartilage. For instance, chondrocytes isolated from the middle and deep zones readily produce an aggrecan-rich matrix to a greater extent than those near the articular surface (49). However, cells in the superficial-most layer synthesize and secrete proteoglycan 4 (PRG4), a contributor to the boundary lubrication function of cartilage $(50,51)$. Stratified tissues have been engineered using chondrocyte subpopulations isolated from different zones and layered through sequential seeding or hydrogel encapsulation (52-54). On the other hand, constructs created from mixed subpopulations of chondrocytes at high density have also exhibited a propensity to develop a zonal architecture resembling native tissue through a process independent of cell sorting (55). It remains unclear how such organization arises but may result from differences in physical constraints or the availability of biochemical factors and nutrients between the surface layer and the deeper tissue.

The formation of a calcified cartilage interface has been another target of recent tissue engineering efforts. The presence of this feature, which anchors the cartilage to the subchondral bone and assists in load distribution, may be pivotal in strengthening the integration of engineered cartilage with bone. Conceptually, the calcification of the interface could be engineered in osteochondral constructs in vitro or induced in vivo following implantation of an engineered chondral or osteochondral graft. The feasibility of the first approach has been shown by researchers using deep zone chondrocytes and culturing them on top of a calcium polyphosphate scaffold in the presence of beta-glycerophosphate to promote calcification (56). This technique also makes use of phenotypic differences in chondrocyte subpopulations, since deep zone chondrocytes are known to produce markers of hypertrophy and calcification such as collagen type $X$ and alkaline phosphatase (57). The in vivo formation of a calcified cartilage interface, as indicated by the presence of a tidemark, has also been observed following the implantation of tissue engineered constructs in rabbit models of osteochondral repair $(58,59)$. However, the appearance of this feature was inconsistent, and the ability to manipulate the in vivo calcification of the cartilage-bone interface has not yet been demonstrated.

\section{THE DEVELOPMENT OF FORM}

Regulation of joint morphology. The process of joint morphogenesis begins around the time of cavitation and rapidly produces congruent articular surfaces with specialized forms. The diversity of these forms is evident from the many types of joints and their associated ranges of motion, from the ball and socket configuration of the hip to the hinge-like knee with dual condyles. The development of the hip in humans is a particularly well documented process. Cavitation occurs around the 7 th week of gestation, and in the subsequent 4 weeks the predominant forms of the spherical femoral head, short femoral neck, and primitive greater trochanter become apparent (60). It is evident that specific patterns of differentiation and growth provide the means of such morphogenetic changes. The processes of growth (i.e., matrix deposition and cell division and hypertrophy) can serve as morphogenetic factors only if they occur differentially throughout a tissue (7). However, the mechanisms which guide these patterns of growth to produce such different forms warrant further study. Recent evidence suggests that specific patterns of growth or differentiation factor 5 and cell division direct morphogenetic changes in the embryonic chick interphalangeal joint (3). Additionally, the role of the local biomechanical environment in the morphogenesis of joints has long been of interest, and mechanical stimuli have been deemed crucial in the proper development of joint form. Paralysis of chick embryos may result in the failure of cavitation at joints sites or the fusion of cartilage anlagen already separated $(61,62)$.

Although morphogenesis results in the development of basic joint forms during embryogenesis, the actual morphology of the joint may continue to change beyond the embryonic 
stage as a result of growth or remodeling. Fetal joints are not proportionately scaled-down versions of their mature counterparts (see images in Fig. 2). Rather, the proportions of joint features may change relative to one another in a manner dependent on the rates and directions of growth. Models of cartilage growth and ossification as mechanically-dependent processes have illustrated how distinct profiles of spatially varying growth rates may alter subsequent joint morphology $(63,64)$. Experimental evidence confirms that postnatal mechanical loading of joints may influence their morphology. Exercise may result in cartilage thickening and bone remodeling to improve congruency in the knees of immature dogs (40). Likewise, exercise can produce cartilage thickening and longer, flatter subchondral surfaces in the femoral heads of immature mice (65).

Biomechanics also plays a pivotal role in the development and treatment of common disorders of joint morphology. Developmental dysplasia of the hip (DDH) is characterized by abnormal growth and morphology of the femoral head and acetabulum. Risk factors for DDH include those linked to genetics (e.g., gender, ethnicity, and family history) and an altered mechanical environment (e.g., breech presentation and oligohydramnios) (66). Dislocation or lateral subluxation of a morphologically normal hip and the resulting alteration in contact stresses may induce dysplasia in the femoral head and acetabulum (67). Nonsurgical treatment for DDH may rely on harnesses or casts to provide reduction of the femoral head within the acetabulum to achieve a mechanical environment which facilitates molding of the articulating surfaces $(68,69)$. Similarly, mechanical manipulation of joint morphology through casting has been used in the treatment of club foot and shown to alter growth rates of cartilage anlagen during this corrective process (70). These findings indicate that biomechanical regulation of cartilage growth continues to be an important factor in determining joint morphology beyond the embryonic stage.

The role of proper form in joint repair. The congruity of apposing joint surfaces is critical to the cartilage's ability to distribute load and provide smooth articulation. Consequently, the restoration of physiologic joint geometry should be an important consideration in therapeutic strategies employing engineered cartilage. The effects of using improperly sized and shaped engineered tissues may resemble those produced by poorly inserted osteochondral grafts. Malpositioned osteochondral grafts may result in abnormal biomechanics including increased contact pressures (71) and cartilage strains (72). To minimize incongruity produced by osteochondral allograft transplantation, orthopedic surgeons frequently use orthotopic grafts from donor tissue matched to the recipient based on size $(73,74)$. Similar care in designing the sizes and shapes of engineered chondral and osteochondral grafts may well be a factor in successful cartilage repair.

Engineering cartilage forms. Chondral tissues of specific shapes and sizes have been generated using various scaffoldbased and scaffold-free techniques. Scaffolds for cartilage tissue engineering may be given an initial shape via casting, free-form fabrication, and similar methods before seeding with cells. The application of these techniques in creating osteochondral constructs resembling a phalanx and mandibular condyle have been demonstrated $(75,76)$. Hydrogels with encapsulated cells are particularly well suited for the creation of constructs with complex shapes, such as those of the patella and mandibular condyle, since they can be polymerized within a negative mold $(77,78)$. In a scaffold-free approach, forms resembling the femoral head, acetabular, or mensical cartilages have been generated by high density seeding of primary (79) or alginate-recovered cells (manuscript in preparation) in negative molds. One concern regarding these techniques is that they only set the initial form of a construct which may change through subsequent growth and remodeling. It may be necessary to predict how such growth would alter the shape of these tissues, perhaps through improvements of the growth models previously described, or to implement measures to control and direct the shape of these tissues during culture.

Just as the morphology of a growing joint is influenced by physical forces in vivo, the shapes of chondral tissues may also be altered via mechanical stimuli in vitro. Although the use of mechanical stimuli to promote functional maturation of engineered cartilage has been an active area of study over the last decade (80), the application of such stimuli to selectively alter the form of cartilaginous tissues is relatively underexplored. Recent work has demonstrated that the application of static bending to immature cartilage explants in a deformationcontrolled manner can alter the shape of these tissues during culture (81). The process by which this reshaping occurred was largely independent of chondrocyte biosynthesis, suggesting the involvement of a biophysical or biochemical remodeling of the extracellular matrix rather than growth. Combining this mechanical reshaping technique with one of the methods which provide initial form may emerge as a complementary approach to engineering shaped chondral tissues.

\section{CONCLUSIONS}

Growth and maturation of cartilage of articular joints are dynamic processes mediated by cell fates and extracellular matrix metabolism. These processes are regulated by complex biochemical and mechanical stimuli which are being elucidated through both in vivo and in vitro approaches. Mathematical models also provide a means of quantitatively describing the changing composition, functional properties, size and shape of tissues, and may be a useful tool for designing experimental conditions to improve engineered tissues. Many lingering questions about the mechanisms and regulation of cartilage growth remain to be answered, and many challenges still exist for the bioengineer trying to create engineered cartilage of a specified size, shape, and maturity. Integrating various fabrication and culture techniques, such as those mentioned here, will become important to meet the clinical requirements for engineered cartilage tissues.

Acknowledgments. We thank Dr. Kyle D. Jadin and Dr. Anna Asanbaeva for discussions and assistance with figures, and Dr. Amanda K. Williamson for providing photographs used in Figure 2. 


\section{REFERENCES}

1. Cole BJ, Malek MM 2004 Articular Cartilage Lesions: A Practical Guide to Assessment and Treatment. New York: Springer, p 336

2. Shum L, Coleman CM, Hatakeyama Y, Tuan RS 2003 Morphogenesis and dysmorphogenesis of the appendicular skeleton. Birth Defects Res C Embryo Today 69:102-122

3. Pacifici M, Koyama E, Iwamoto M 2005 Mechanisms of synovial joint and articular cartilage formation: recent advances, but many lingering mysteries. Birth Defects Res C Embryo Today 75:237-248

4. Khan IM, Redman SN, Williams R, Dowthwaite GP, Oldfield SF, Archer CW 2007 The development of synovial joints. Curr Top Dev Biol 79:1-36

5. Klisch SM, Chen SS, Sah RL, Hoger A 2003 A growth mixture theory for cartilage with applications to growth-related experiments on cartilage explants. J Biomech Eng 125:169-179

6. Klisch SM, Asanbaeva A, Oungoulian SR, Masuda K, Thonar EJ, Davol A, Sah RL. A cartilage growth mixture model with collagen remodeling: validation protocols $\mathrm{J}$ Biomech Eng, in press

7. Thorogood P 1983 Morphogenesis of cartilage. In: Hall B (ed) Cartilage. New York: Academic Press, pp 223-254

8. Mankin HJ, Mow VC, Buckwalter JA, Iannotti JP, Ratcliffe A 1994 Form and function of articular cartilage. In: Simon SR (ed) Orthopaedic Basic Science. Rosemont, IL: American Academy of Orthopaedic Surgeons, pp 1-44

9. Jadin KD, Wong BL, Bae WC, Li KW, Williamson AK, Schumacher BL, Price JH, Sah RL 2005 Depth-varying density and organization of chondrocyte in immature and mature bovine articular cartilage assessed by 3-D imaging and analysis. J Histochem Cytochem 53:1109-1119

10. Skalak R, Gasgupta G, Moss M, Otten E, Dullemeijer P, Vilmann H 1982 Analytical description of growth. J Theor Biol 94:555-577

11. Klisch SM, Van Dyke T, Hoger A 2001 A theory of volumetric growth for compressible elastic materials. Math Mech Solids 6:551-575

12. Mankin HJ 1962 Localization of tritiated thymidine in articular cartilage of rabbits I. Growth in immature cartilage. J Bone Joint Surg Am 44:682-698

13. Apte SS 1990 Validation of bromodeoxyuridine immunohistochemistry for localization of S-phase cells in decalcified tissues. A comparative study with tritiated thymidine autoradiography. Histochem J 22:401-408

14. Kavanagh E 2002 Division and death of cells in developing synovial joints and long bones. Cell Biol Int 26:679-688

15. Hayes AJ, MacPherson S, Morrison H, Dowthwaite G, Archer CW 2001 The development of articular cartilage: evidence for an appositional growth mechanism. Anat Embryol (Berl) 203:469-479

16. Dowthwaite GP, Bishop JC, Redman SN, Khan IM, Rooney P, Evans DJ, Haughton L, Bayram Z, Boyer S, Thompson B, Wolfe MS, Archer CW 2004 The surface of articular cartilage contains a progenitor cell population. J Cell Sci 117:889-897

17. Alsalameh S, Amin R, Gemba T, Lotz M 2004 Identification of mesenchymal progenitor cells in normal and osteoarthritic human articular cartilage. Arthritis Rheum 50:1522-1532

18. Fickert S, Fiedler J, Brenner RE 2004 Identification of subpopulations with characterisitics of mesenchymal progenitor cells from human osteoarthritic cartilage using triple staining for cell surface markers. Arthritis Res Ther 6:R422-R432

19. Wilsman NJ, Farnum CE, Leiferman EM, Fry M, Barreto C 1996 Differential growth by growth plates as a function of multiple parameters of chondrocytic kinetics. J Orthop Res 14:927-936

20. Aydelotte MB, Kuettner KE 1988 Differences between sub-populations of cultured bovine articular chondrocytes. I. Morphology and cartilage matrix production. Connect Tissue Res 18:205-222

21. Williamson AK, Chen AC, Sah RL 2001 Compressive properties and functioncomposition relationships of developing bovine articular cartilage. J Orthop Res 19:1113-1121

22. Williamson AK, Chen AC, Masuda K, Thonar EJ, Sah RL 2003 Tensile mechanical properties of bovine articular cartilage: variations with growth and relationships to collagen network components. J Orthop Res 21:872-880

23. Eyre DR, Koob TJ, Van Ness KP 1984 Quantitation of hydroxypyridinium crosslinks in collagen by high-performance liquid chromatography. Anal Biochem 137:380-388

24. Thonar EJ, Buckwalter JA, Kuettner KE 1986 Maturation-related differences in the structure and composition of proteoglycans synthesized by chondrocytes from bovine articular cartilage. J Biol Chem 261:2467-2474

25. Jadin KD, Bae WC, Schumacher BL, Sah RL 2007 Three-dimensional (3-D) imaging of chondrocytes in articular cartilage: growth-associated changes in cell organization. Biomaterials 28:230-239

26. Hunziker EB, Kapfinger E, Geiss J 2007 The structural architecture of adult mammalian articular cartilage evolves by a synchronized process of tissue resorption and neoformation during postnatal development. Osteoarthritis Cartilage 15:403413

27. Asanbaeva A, Masuda K, Thonar EJ, Klisch SM, Sah RL 2007 Mechanisms of cartilage growth: modulation of balance between proteoglycan and collagen in vitro using chondroitinase ABC. Arthritis Rheum 56:188-198

28. Klein TJ, Chaudhry M, Bae WC, Sah RL 2007 Depth-dependent biomechanical and biochemical properties of fetal, newborn, and tissue-engineered articular cartilage. J Biomech 40:182-190

29. Maroudas A 1979 Physico-chemical properties of articular cartilage. In: Freeman MAR (ed) Adult Articular Cartilage. Tunbridge Wells, England: Pitman Medical, pp 215-290

30. Hunziker EB, Michel M, Studer D 1997 Ultrastructure of adult human articular cartilage matrix after cryotechnical processing. Microsc Res Tech 37:271-284
31. Schinagl RM, Gurskis D, Chen AC, Sah RL 1997 Depth-dependent confined compression modulus of full-thickness bovine articular cartilage. J Orthop Res 15:499-506

32. Kempson GE, Freeman MA, Swanson SA 1968 Tensile properties of articular cartilage. Nature 220:1127-1128

33. Davol A, Bingham MS, Sah RL, Klisch SM. A nonlinear finite element model of cartilage growth. Biomech Model Mechanobiol, in press. DOI: 10.1007/s10237-0070098-6

34. Luyten FP, Hascall VC, Nissley SP, Morales TI, Reddi AH 1988 Insulin-like growth factors maintain steady-state metabolism of proteoglycans in bovine articular cartilage explants. Arch Biochem Biophys 267:416-425

35. Sah RL, Kim YJ, Doong JH, Grodzinsky AJ, Plaas AH, Sandy JD 1989 Biosynthetic response of cartilage explants to dynamic compression. J Orthop Res 7:619-636

36. Hascall VC, Luyten FP, Plaas AH, Sandy JD 1990 Steady-state metabolism of proteoglycans in bovine articular cartilage. In: Maroudas A, Kuettner K (eds) Methods in Cartilage Research. San Diego, CA: Academic Press, pp 108-112

37. Ahsan T, Harwood FL, McGowan KB, Amiel D, Sah RL 2005 Kinetics of collagen crosslinking in adult bovine articular cartilage. Osteoarthritis Cartilage 13:709-715

38. DiMicco MA, Sah RL 2003 Dependence of cartilage matrix composition on biosynthesis, diffusion, and reaction. Transport Porous Med 50:57-73

39. Klein TJ, Sah RL 2007 Modulation of depth-dependent properties in tissueengineered cartilage with a semi-permeable membrane and perfusion: a continuum model of matrix metabolism and transport. Biomech Model Mechanobiol 6:21-32

40. Helminen HJ, Hyttinen MM, Lammi MJ, Arokoski JP, Lapvetelainen T, Jurvelin J, Kiviranta I, Tammi MI 2000 Regular joint loading in youth assists in the establishment and strengthening of the collagen network of articular cartilage and contributes to the prevention of osteoarthrosis later in life: a hypothesis. J Bone Miner Metab $18: 245-257$

41. Asanbaeva A, Masuda K, Thonar EJ, Klisch SM, Sah RL 2008 Cartilage growth and remodeling: modulation of balance between proteoglycan and collagen in vitro with beta-aminopropionitrile. Osteoarthritis Cartilage 16:1-11

42. Asanbaeva A, Masuda K, Thonar EJ, Klisch SM, Sah RL. Regulation of immature cartilage growth by IGF-I, TGF-alpha 1, BMP-7, and PDGF-AB: role of metabolic balance between fixed charge and collagen network. Biomech Model Mechanobiol, in press. DOI: 10.1007/s10237-007-0096-8

43. Guilak F, Sah RL, Setton LA 1997 Physical regulation of cartilage metabolism. In Mow VC, Hayes WC (eds) Basic Orthopaedic Biomechanics. New York: Raven Press, pp 179-207

44. Lima EG, Bian L, Mauck RL, Byers BA, Tuan RS, Ateshian GA, Hung CT 2006 The effect of applied compressive loading on tissue-engineered cartilage constructs cultured with TGF-beta3. Conf Proc IEEE Eng Med Biol Soc 1:779-782

45. Freed LE, Langer R, Martin I, Pellis NR, Vunjak-Novakovic G 1997 Tissue engineering of cartilage in space. Proc Natl Acad Sci USA 94:13885-13890

46. Buschmann MD, Gluzband YA, Grodzinsky AJ 1995 Mechanical compression modulates matrix biosynthesis in chondrocyte/agarose culture. J Cell Sci 108:14971508

47. Knight MM, Lee DA, Bader DL 1998 The influence of elaborated pericellular matrix on the deformation of isolated articular chondrocytes cultured in agarose. Biochim Biophys Acta 1405:67-77

48. Alsberg E, Anderson KW, Albeiruti A, Rowley JA, Mooney DJ 2002 Engineering growing tissues. Proc Natl Acad Sci USA 99:12025-12030

49. Aydelotte MB, Greenhill RR, Kuettner KE 1988 Differences between subpopulations of cultured bovine articular chondrocytes. II. Proteoglycan metabolism. Connect Tissue Res 18:223-234

50. Schumacher BL, Block JA, Schmid TM, Aydelotte MB, Kuettner KE 1994 A nove proteoglycan synthesized and secreted by chondrocytes of the superficial zone of articular cartilage. Arch Biochem Biophys 311:144-152

51. Schmidt TA, Gastelum NS, Nguyen QT, Schumacher BL, Sah RL 2007 Boundary lubrication of articular cartilage: role of synovial fluid constituents. Arthritis Rheum $56: 882-891$

52. Klein TJ, Schumacher BL, Schmidt TA, Li KW, Voegtline MS, Masuda K, Thona EJ, Sah RL 2003 Tissue engineering of stratified articular cartilage from chondrocyte subpopulations. Osteoarthr Cartil 11:595-602

53. Klein TJ, Schumacher BL, Blewis ME, Schmidt TA, Voegtline MS, Thonar EJ, Masuda K, Sah RL 2006 Tailoring secretion of proteoglycan 4 (PRG4) in tissueengineered cartilage. Tissue Eng 12:1429-1439

54. Sharma B, Williams CG, Kim TK, Sun D, Malik A, Khan M, Leong K, Elisseeff JH 2007 Designing zonal organization into tissue-engineered cartilage. Tissue Eng 13:405-414

55. Hayes AJ, Hall A, Brown L, Tubo R, Caterson B 2007 Macromolecular organization and in vitro growth characteristics of scaffold-free neocartilage grafts. J Histochem Cytochem 55:853-866

56. Allan KS, Pilliar RM, Wang J, Grynpas MD, Kandel RA 2007 Formation of biphasic constructs containing cartilage with a calcified zone interface. Tissue Eng 13:167177

57. Sun Y, Kandel R 1999 Deep zone articular chondrocytes in vitro express genes that show specific changes with mineralization. J Bone Miner Res 14:1916-1925

58. Schaefer D, Martin I, Jundt G, Seidel J, Heberer M, Grodzinsky A, Bergin I, Vunjak-Novakovic G, Freed LE 2002 Tissue-engineered composites for the repair of large osteochondral defects. Arthritis Rheum 46:2524-2534

59. Tanaka T, Komaki H, Chazono M, Fujii K 2005 Use of a biphasic graft constructed with chondrocytes overlying a beta-tricalcium phosphate block in the treatment of rabbit osteochondral defects. Tissue Eng 11:331-339

60. Lee MC, Eberson CP 2006 Growth and development of the child's hip. Orthop Clin North Am 37:119-132 
61. Drachman DB, Sokoloff L 1966 The role of movement in embryonic joint development. Dev Biol 14:401-420

62. Mitrovic D 1982 Development of the articular cavity in paralyzed chick embryos and in chick embryo limb buds cultured on chorioallantoic membranes. Acta Anat (Basel) 113:313-324

63. Heegaard JH, Beaupre GS, Carter DR 1999 Mechanically modulated cartilage growth may regulate joint surface morphogenesis. J Orthop Res 17:509-517

64. Shefelbine SJ, Carter DR 2004 Mechanobiological predictions of growth front morphology in developmental hip dysplasia. J Orthop Res 22:346-352

65. Plochocki JH, Riscigno CJ, Garcia M 2006 Functional adaptation of the femoral head to voluntary exercise. Anat Rec A Discov Mol Cell Evol Biol 288:776-781

66. Shapiro F 2001 Pediatric Orthopedic Deformities. San Diego: Academic Press, p 953

67. Siffert RS 1981 Patterns of deformity of the developing hip. Clin Orthop Relat Res 14-29

68. Gabuzda GM, Renshaw TS 1992 Reduction of congenital dislocation of the hip. J Bone Joint Surg Am 74:624-631

69. Storer SK, Skaggs DL 2006 Developmental dysplasia of the hip. Am Fam Physician 74:1310-1316

70. Brand RA, Siegler S, Pirani S, Morrison WB, Udupa JK 2006 Cartilage anlagen adapt in response to static deformation. Med Hypotheses 66:653-659

71. Koh JL, Wirsing K, Lautenschlager E, Zhang LO 2004 The effect of graft height mismatch on contact pressure following osteochondral grafting. A biomechanical study. Am J Sports Med 32:317-320

72. Wu JZ, Herzog W, Hasler EM 2002 Inadequate placement of osteochondral plugs may induce abnormal stress-strain distributions in articular cartilage-finite element simulations. Med Eng Phys 24:85-97
73. Convery FR, Meyers MH, Akeson WH 1991 Fresh osteochondral allografting of the femoral condyle. Clin Orthop Relat Res 273:139-145

74. Bugbee WD 2004 Osteochondral allograft transplantation. In: Cole BJ, Malek MM (eds) Articular Cartilage Lesions: A Practical Guide to Assessment and Treatment. New York: Springer, pp 82-94

75. Sedrakyan S, Zhou ZY, Perin L, Leach K, Mooney D, Kim TH 2006 Tissue engineering of a small hand phalanx with a porously casted polylactic acidpolyglycolic acid copolymer. Tissue Eng 12:2675-2683

76. Schek RM, Taboas JM, Hollister SJ, Krebsbach PH 2005 Tissue engineering osteochondral implants for temporomandibular joint repair. Orthod Craniofac Res 8:313-319

77. Hung CT, Lima EG, Mauck RL, Taki E, LeRoux MA, Lu HH, Stark RG, Guo XE, Ateshian GA 2003 Anatomically shaped osteochondral constructs for articular cartilage repair. J Biomech 36:1853-1864

78. Alhadlaq A, Mao JJ 2005 Tissue-engineered osteochondral constructs in the shape of an articular condyle. J Bone Joint Surg Am 87:936-944

79. Aufderheide AC, Athanasiou KA 2007 Assessment of a bovine co-culture, scaffoldfree method for growing meniscus-shaped constructs. Tissue Eng 13:2195-2205

80. Guilak F, Setton LA 2003 Functional tissue engineering and the role of biomechanical signaling in the articular cartilage repair. In: Guilak F, Butler D, Mooney D, Goldstein S (eds) Functional Tissue Engineering. New York: Springer-Verlag, pp 277-290

81. Williams GM, Lin JW, Sah RL 2007 Cartilage reshaping via in vitro mechanical loading. Tissue Eng 13:2903-2911 